\title{
DEVELOPMENT OF CRITIQUING SYSTEMS IN NETWORK ORGANIZATIONS
}

\author{
Ola Leifler ${ }^{1}$, Björn Johansson ${ }^{1}$, Mats Persson ${ }^{2} \&$ Georgios Rigas $^{2}$ \\ ${ }^{1}$ Linköping University, Sweden, ${ }^{2}$ National Defence College, Sweden
}

\begin{abstract}
Recently, network organizations have been suggested as a solution for future crisis management and warfare. This will, however, have consequences for the development of decision support and critiquing systems. This paper suggests that there are special conditions that need to be taken into account when providing the means for decision-making in networked organizations. Hence, three research problems are suggested that need to be investigated in order to develop useful critiquing systems for future command and control systems.
\end{abstract}

Key words: decision support, critiquing systems, crisis management

\section{INTRODUCTION}

The nature of decision support is bound to change with the new types of network-centric organizations for military command and control that are emerging. When responsibilities are delegated to a larger extent and organizations need to adapt more and more rapidly to changes in the environment, the need for rapid and well-informed decisions increases. There has also been a great increase in precision and strike force in the military sector (van Creveld, 2000), meaning that each decision is likely to have much greater consequences than earlier. Thus, at the same time that consequences and risks are increasing, more people are supposed to make decisions and take action in parallel. This is to be done under time pressure and without errors. This, in turn, calls for a new view of decision support as a coordination tool and as a tool for collaborative work, supporting decisions 
at a number of organizational levels and in specialist functions, rather than the older expert-system, single decision-maker, view.

In 1988, Kraemer and King presented a survey of the development of group decision support systems in the United States (Kraemer and King, 1988). Although many items they described as decision support (shared databases, intranets, shared displays) are everyday technology now, the problems of building decision support systems for teams or groups seem to be as valid as they were 15 years ago (Kraemer and King, 1988, p. 369):

The technical systems necessary to create effective GDSS tools for realtime decision-making are difficult to build, and the potential uses of such systems are not well specified. The most coherent specifications of the decision process are built on the rational model of decision making, which at best accounts for only a part of the true decision-making behavior that takes place in group decision making.

Although the field of decision-making clearly has made progress with fields like Dynamic Decision Making and Naturalistic Decision Making, their observations still hold, something that we will elaborate on later in this paper. Even if research has given a deepened understanding of the nature of decision making, there is an evident need for a collaborative or distributed view on decision making in the discussion of future decision support systems, otherwise we might end up with products based on assumptions that are no longer valid in contemporary work environments.

The authors of this paper are all researchers within a Swedish research project aiming at developing a mobile command and control concept for the future network-based defense (see Section 2). One important part of this project is to evaluate and develop a critiquing system (Sundin and Friman, 1998) for the staff. As a point of departure for this work, we will analyze the problems of current decision support technology in connection with team decision-making and networked organization structures.

\section{ROLF 2010}

Organizations such as the rescue services or military defense have traditionally been hierarchically structured. As such, the command and control function has been located in the uppermost hierarchical level. Today it is argued that current hierarchical organizations are too rigid to be able to act and react on situations in future and highly dynamic environments. As an option for handling the dynamics, so called "network organizations" have been proposed as a solution. The structure of network organizations is 
considered a possible answer to several problems of exerting command and control among military communities (Alberts et al., 2000; Cebrowski and Garstka, 1998; Sundin and Friman, 2000).

Implementations of such an organizational structure imply that traditional hierarchical levels of command could be flattened out, reduced or even completely removed. Reducing levels of command is considered advantageous and necessary to shorten the reaction time to changes in the environment since data processing within every level of command is considered time consuming and thereby would seriously hamper any necessary action. Furthermore, it is assumed that if a decision-maker is provided with enough data presented in an understandable way he or she will be able to make "optimal" decisions. Consequently, larger amounts of data have to be handled by the commanders within network organizations in comparison with traditional hierarchical organizations.

In Sweden, a new command and control concept is currently under development. The project, known as ROLF 2010 (Joint Mobile Command and Control Function), is aimed at creating a staff environment where a team of decision-makers can work jointly (see Figure 1). With the aid of information technology in the form of shared workspaces and direct access to sensor information, it is envisioned that commanders will be able to make swift and correct decisions. However, greater demands will also be made on mission control centers such as the ROLF 2010 staff unit, since "[...] it will require that the commander and his staff will be able to handle greater amounts of information and greater complexity than before" (Sundin and Friman, 2000).

Naturally, a very important part of such a system is the tools used by the commanders in the decision process. In the ROLF-vision, a critiquing system, rather than a traditional decision support system, is a central part.

In a network-centric organization, analyzing large amounts of data for the decision maker is of course an important issue, but what is really new is the amount of coordination that the decision support systems have to be both aware of, and actively supporting.

By "being aware of" coordination, we refer to the fact that the internal model of the decision-making process, used by the decision-support system, has to take into account the concurrent actions taken by all human members that the system is supposed to interact with. That is, we cannot be satisfied with having a decision-support system that is only aware of the local actions performed by the human operator it is currently interacting with. 


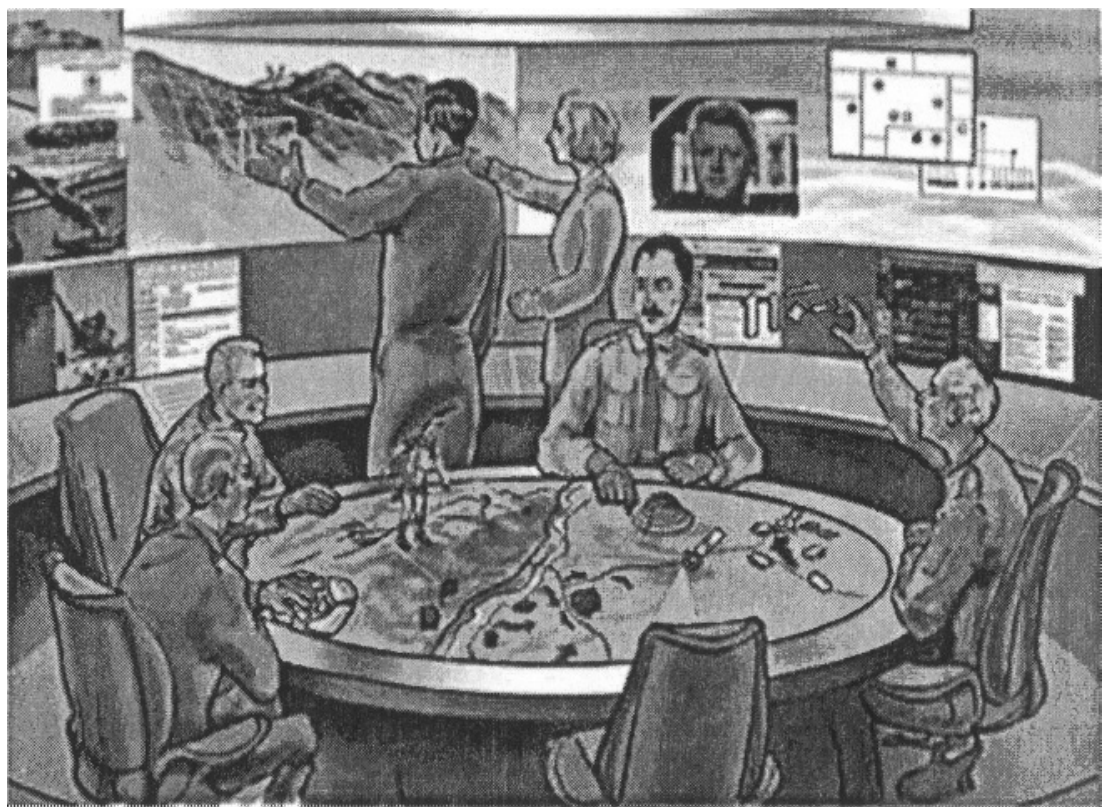

Figure 1. An artists view of the future ROLF 2010 work environment.

By "actively supporting" coordination, we refer to the fact that the system would have to provide more than only a means of communication, although such functionalities were traditionally regarded as decision support systems (see Section 1). We believe that analyzing user input and providing comments and feedback on such input would, in a network-centric organization, have to be done with groups of people providing the input and also receiving responses. This can be seen in contrast to traditional systems for intelligent decision support that have relied on a simple use case where a single user is provided with expert advice from a computer systems that analyzes a user-defined solution for a given problem, with respect to criteria defined by human domain experts.

\section{INTELLIGENT DECISION SUPPORT}

The idea of decision support is to aid a single or several decision-makers in situations where there is either too much, too incomprehensive information or the consequence of the decision is of such great importance that even trivial problems need some guidance to ensure a correct decision. Since humans are very good in their comprehension of situations but have a limited capability for analyzing, computers seem to be a suitable aid for that task. 
Decision support can be manifested in a number of ways. First, a user could explicitly ask an expert system for a solution to a problem. This kind of decision support has been successful to some extent within areas where systems are "closed", meaning that the influences from external, unforeseen sources are minimal. Another kind of approach is the "critiquing" where the user presents a proposal for action, and is given feedback on that proposal. Such a system aims at three things (Silverman, 1992):

- Recognizing and analyzing human erroneous action;

- Giving persuasive feedback, and

- Adapting to the situation and previous experience.

Given that several individuals making decisions jointly will operate future command and control situations, critiquing systems emerge as being of great interest. As opposed to suggesting "solutions" to whatever problem is at hand for a group of decision-makers, critiquing could mean that the group members are notified of specific actions taken by others, actions that might in turn have an effect on their own work. This kind of feedback mechanism could, we believe, serve as a useful platform for constructing new kinds of intelligent decision support. However, what does it mean to provide "intelligent decision support" for a group of people collaborating on some task?

\section{TEAMWORK}

Irrespective of the general task at hand for the personnel exerting command and control in a ROLF 2010 like environment, the members typically operate as a team. To distinguish what constitutes and distinguishes a team from a group we have agreed upon Cannon-Bowers', Salas' and Converse's definition of a team as (Cannon-Bowers et al., 1993):

[T]wo or more individuals who must interact cooperatively and adoptively in pursuit of shared valued objectives. Further, team members have clearly defined differentiated roles and responsibilities, hold taskrelevant knowledge, and are interdependent.

The necessity for interdependency could be exemplified by Brannick and Prince's (Brannick and Prince, 1997) statement where they stress that one of the central features of teamwork is coordination where there is some kind of adjustment that the team members make in order to reach the goal.

Given that the team will carry out its task in an uncertain environment, the primary components of a decision-support system to develop are those which help to reduce that uncertainty. However, as Galegher points out, decision making does not require only gathering data about the surroundings 
(Galegher, 1990). It also comprises the interpretation of the problems at hand, the definition of (sub)goals and strategies and effectively being able to make representations of the decisions to internal and external constituencies.

Being able to design appropriate and efficient solutions implies an understanding of how team decision-making is carried out. This is however not easy and to illustrate what difficulties designers may encounter Galegher writes (Galegher, 1990, p. 8):

Real-world decision making, however, is typically not well specified, stable, or orderly enough to permit decision makers to understand their situation and consciously adopt a suitable problem solving approach. Rather, problems may contain elements of both uncertainty and equivocality, and are likely to present themselves at unpredictable times. Moreover, groups may lack awareness of the type of decision in which they are embroiled.

The uses of technology to support decision teams are problematic from other view-points as well. The implementation of command posts such as

ROLF 2010 could easily encourage centralizing command and control.

However, as van Creveld puts it (van Creveld, 2000):

The more centralized the system, the greater the danger that it will be paralyzed if enemy action causes the directing brain to be eliminated or communications with it to be impaired.

This important statement gives cause for reconsidering novel organization forms and command structures on the one hand, as well as directives on how decision teams should operate on the other.

Focusing on the latter will most likely imply new work methods among the team members than those traditionally used. Furthermore, it is possible to commit the decision team to support the organization as a customer service by providing necessary information to different organizational parts when needed. However, when it comes to organizational changes where traditional hierarchies are shifted to post-bureaucratic, or network, organizations a problem of responsibility for made decisions appears. There have been successful attempts to reduce some of the side effects of hierarchies, but decision processes are still hierarchical in nature (Galbraith, 1993). An important issue is therefore how to support decision teams in determining when a delegation of mandate and responsibilities to other levels of command can be appropriate or not.

Computer support at the group level has traditionally only focused on providing means of communication and making certain aspects of group coordination explicit (Schmidt and Bannon, 1992). On the other hand, much more has been done to support individual decision makers, where there have 
been several military projects involved. Since we are more interested in systems that try to "understand" the actions taken by the user rather than just providing a simple means of book-keeping and communication, we will restrict ourselves to Critiquing Systems.

\section{CURRENT CRITIQUING SYSTEMS}

\subsection{Traditional critiquing}

When constructing a critiquing system, it differs slightly from the design of a more traditional expert system in that the system expects a user to provide a solution to a given type of problem. This solution is then either compared to what the system itself would have suggested with its built-in background knowledge or analyzed with respect to predefined rules for constructing a solution and the metrics for evaluating them, also through domain knowledge provided by a domain expert.

Typically, the user scenario for a critiquing system is that a few experts on a certain topic are consulted to provide information about the standard operating guidelines for solving problems in the domain, common pitfalls, measures of success and other kinds of information of use to a system that should monitor and evaluate the performance of users. Users are then assumed not to be experts in the domain, yet sufficiently apt to propose somewhat correct solutions to problems that may arise in this domain and also proficient enough to understand criticism regarding their performance, if appropriately motivated. The user scenario, when knowledge has been entered and verified by the domain experts, is that a single user enters information (most often in written form) describing a suggestion to solve a specific problem. The kind of problems studied in military contexts have often been in the form of a classical planning problem where a clearly defined goal is to be reached through the use of some available resources under certain constraints (time-constraints or other).

Such well-defined problems are less common in real life - there are not always metrics that can be used to evaluate performance in a way that humans are not capable of doing better themselves. For instance, the reliability of information can best be evaluated by asking intelligence officers responsible, so no easy automatic verification mechanism would be readily available. Thus, the commonly accepted preconditions for critiquing systems are not likely to be met in real operating conditions. 


\subsection{Formulating plans as a user}

In the case of helping a user to formulate a military plan according to a formal description of a plan (most often in the "Course of Action" formalism for military plans (US Army, 1997)) Kott et al. used a top-down approach in a project centered around the CADET (Kott et al., 2002; Group, 2003) tool. In that project, plans were given a sketch-like description at first and later made more concrete as they were described in terms of how they should be achieved. The narrowing of goals was supposed to guide the user to provide all the necessary information so as to produce at least a complete description of the plan. When providing this guidance for the user, CADET informed the user of available resources but was not overly strict about constraint satisfaction in every step, much like a word-processor that actually allows you to mistype since it is much faster to correct errors afterwards. The time constraints for the whole operation were shown in a matrix describing the interdependencies between different parts.

Previously in similar projects - that is, in projects that had the user specify a plan according to a top-down approach - the guidance for the user was often conducted so that only syntactically correct plans could be constructed as a result of the process. The syntactical control consisted of, for instance, making sure that descriptions of "how to achieve goals" were made increasingly more specific. The user was supposed to progress along the branches of the "tree" formed by having the "root" node as being your main intention with the plan and the children to a node being the divisions of a task into more manageable units. For example the INSPECT/EXPECT project (ISI, 2003; Valente et al., 1996) consisted of producing a verification tool for plans constructed with the Air Campaign Planning Tool used by the US Air Force. The verification tool could insure that basic constraints in such a hierarchical structure were fulfilled, such as the fact that nodes should either be a root node or have a parent, and either be leaves or have children. That is, a task would have to be decomposed into parts until some predefined level was reached.

\section{DISCUSSION}

There are several issues with previous approaches to decision support when compared to the ROLF 2010-like environments that signal a need to renew our perceptions of how critiquing should be performed. 


\subsection{Problems with formal verification}

One of the problems with the formalization of military plans is that, with the development of the ROLF $2010 \mathbf{C}^{2}$ concept, we move in a direction where detailed control over subordinate units is sacrificed in favor of formulating a Commanders Intent as the primary means of exerting command and control. This has the effect of making plans that express this intent in fact less suited for formalization and formal verification and more like guidelines for human interpretation only. The act of verifying if an endstate has been achieved is not trivial if the end-state is in the form of "having control of a region".

In ROLF 2010 there have been studies around the concept and use of strategic optimizing simulations (Woodcock et al., 2003). These simulations have been intended to be used as a part of a decision support system, if provided with a critiquing system that could analyze the output of the simulations. The simulation environment is built on the use of genetic algorithms that evolve over many generations and hopefully converge on an approximate solution to the given problem. Such methods for solving problems have, however, inherent difficulties since it is difficult to understand what the evolutionary process creates. Even though the mechanisms that are used to create new solutions from old ones are fairly simple, there is little hope of understanding why a certain solution has been created instead of another and thus it may not be possible to give constructive criticism that reflects on the reasons for choosing it.

Though the need for verification may persist, it is not at all clear how such verification could be performed when there is so much freedom for the subordinate commanders to implement the given directives as they see fit. However, there may be other ways than merely using formal logics to deduce inconsistencies that can give valuable feedback on what is being planned or, in the case of a subordinate commander, following orders.

\subsection{Decision process in ROLF 2010}

Given the descriptions above, both the one of the decision making process of a team in Section 4 and of the ROLF 2010 environment in Section 2, we can see that there are some special characteristics of the ROLF 2010 environment, compared to other environments that have been fairly wellstudied. We need not worry too much about the execution of our plans, neither need we care about the activation of decisions since we rely on the fact that data are collected and made available to us either by means of technical equipment or by intelligence units. Also, the decision process is one where decisions are constantly being refined and amended and even 
when delivered as orders, they are not very precise regarding what should be done by the subordinate units at hand. So what is actually meant by a certain decision process? Is it a process that applies to each member of a staff? Or should it apply to the whole staff, so that the staff at any given time only is concerned with one of the activities involved in planning? If neither of the above applies, then how does the model actually describe the work process?

The quality of the feedback we can receive from a critiquing system depends heavily on what information is available from the "answer" (a COA plan, output from some kind of simulation software etc.).

If given an answer with little supplementary information as to the motivation for that answer, the corresponding range of possible items to criticize would be enormous for a critiquing system.

The opposite problem also applies for systems that give answers as output. Many simulation-based decision support systems work in this way. The user (or a sensor system or other technical systems) provides input, which is treated by the decision support system. The output given is the decision support, meant to be used as a possible solution to a problem. A decision support system capable of analyzing complex situations perhaps can provide useful output. However, such systems are bound to be so complex and opaque that it is virtually impossible for the user to look "under the hood" of the system and objectively criticize the basis for the advice. In both situations, the part given the answer needs to be informed of how the answer was reached, otherwise it is very difficult to relate to it in an objective way.

\subsection{Criticizing Decisions}

New organizations and new technical systems do not only function as tools, they also fundamentally change the work process, and thus the context of the decision making. Actions that earlier were decided by single individuals may now be the result of several decisions made at different organizational levels. In the process of refining and constantly evolving orders, it is not trivial to identify the actual decision that is to be input to a decision support system. Even if decisions may be recognized as physical documents, their contents may not be defined well enough to allow for automated analysis of correctness.

The discussion above and the analysis of earlier decision support systems leads towards a new way to look at decision support. Earlier, the main function of a decision support system was either to provide a suggestion for a solution to a problem, or to provide criticism on a user suggestion. In modern command and control structures, working in an uncertain context, where it is getting increasingly difficult to identify when and where 
decisions are made and should be made, systems that support coordination are probably more useful than traditional decision support systems. Future decision support has to consider the team perspective.

The most central part of optimizing team performance is coordination. A system that supports all team members and their specific roles, so they both can increase the individual effort and coordinate better, is likely to have a huge impact on the total team performance.

\subsection{Suggested research}

There are three research aspects of intelligent group support that we believe will be crucial to address in future research:

1. It is necessary to investigate if it is possible to create a representation of the activities in the network organization. Such a representation is needed, in real time, if the critiquing system is going to be able to provide feedback on the decisions and actions of different actors. How to collect information, and what information, are central parts of this problem.

2. If a critiquing system is to be used by several actors in an organization, it probably has to be transparent enough for the users to create a common understanding of the system's abilities and limitations. If not, the critiquing system itself is likely to become an impediment for the users. Therefore, we suggest that tests must be performed with low-fidelity simulations, but with real operators, in order to investigate the impact of such a system on organizational performance.

3. In contrast to other domains where critiquing systems have been used, such as medical applications, it is difficult to define what expert knowledge really is. Apart from obvious constraints on when decisions need to be delivered, and which protocols that should be followed, it is not clear how to specify what the quality of actions that a group performs is. Thus, allowing for the group members themselves to produce simple constraints they believe to be useful could give researchers insight into what kind of issues the critiquing system should address. To assess such knowledge is a crucial part in the work of developing a critiquing system. It should, however, be noted that there are some fundamental problems in doing this, especially since there are no such organizations as the ones intended available today, when designing such a critiquing system. Once again, this calls for studies using both simulations of the intended context and professional users. 


\subsection{Final remarks}

In this article, we have analyzed the problem of traditional decision support in relation to the demands made by future organizational structures. We have also suggested three areas that need to be investigated: information gathering, user acceptance and knowledge gathering.

Military decision-making is a field where the consequences of decisions are so great that the subject of decision support hardly can be neglected. This paper has pointed to both the problem of formal verification in decision support systems and the specific problems that arise when trying to support network organizations. Only research efforts taking the actual difficulties and circumstances of collaborative work environments seriously are likely to give useful insights into the design of computer systems for future critiquing systems.

\section{REFERENCES}

Alberts, D. S., Gartska, J. J., and Stein, F. P. (2000). Network Centric Warefare: Developing and Leveraging Information Superiority. National Defense University Press, Washington, DC.

Brannick, M. T. and Prince, C. (1997). An overview of team performance measurement. In Brannick, M. T., Salas, E., and Prince, C., editors, Team performance assessment and measurement: theory, methods, and applications, Series in applied psychology, pages 3Đ16. Lawrence Erlbaum Associates, Mahwah, N.J.

Cannon-Bowers, J. A., Salas, E., and Converse, S. (1993). Shared mental models in expert team decision making. In Castellan, N. J., editor, Individual and group decision making: current issues, pages 221-246. Lawrence Erlbaum Associates, Hillsdale, N.J.

Cebrowski, A. K. and Garstka, J. J. (1998). Network-centric warfare: Its origin and future. U.S.Naval Institute Proceedings, 124(1):28-35.

Galbraith, J. R. (1993). Challenges to the established order. In Galbraith, J. R., Lawler III, E. E., and Associates, editors, Organizing for the Future: The New Logic for Managing Complex Organizations, pages 1-12. Jossey-Bass, San Francisco, Calif.

Galegher, J. (1990), Technology for intellectual teamwork: Perspectives on research and design. In Galegher, J., Kraut, R., and Egido, C., editors, Intellectual Teamwork: Social and Technological Foundations of Cooperative Work, pages 1-20. Lawrence Erlbaum Associates, Hillsdale, New Jersey. http://www.erlbaum.com/60.htm.

Group, L. C. (2003). CADET.

http://www.arl.army.mil/aro/arowash/rt/sbir/99brochure/carnegie.htm.

ISI (2003). EXPECT. http://www.isi.edu/ikcap/expect/.

Kott, A., Ground, L., Budd, R., Rebbapragada, L., and Langston, J. (2002). Toward practical knowledge-based tools for battle planning and scheduling. In Proceedings of the Eighteenth National Conference on Artificial Intelligence, pages 894-899, Edmonton, Alberta, Canada.

Kraemer, K. L. and King, J. L. (1988). Computer-based systems for cooperative work and group decision making. ACM Computing Surveys, 20(2):115Đ146. 
Schmidt, K. and Bannon, L. (1992). Taking CSCW seriously. Supporting Articulation Work, Computer Supported Cooperative Work -An International Journal, 1(1- 2):7-41.

Silverman, B. G. (1992). Critiquing Human Error - A Knowledge Based Human-Computer Collaboration Approach. Academic Press, London.

Sundin, C. and Friman, H., editors (1998). ROLF 2010 - A Mobile Joint Command and Control Concept. Gotab Erlanders.

Sundin, C. and Friman, H., editors (2000). ROLF 2010 - The Way Ahead and The First Step. Gotab Erlanders, Stockholm.

US Army (1997). Field Manual 101-5: Staff Organisation and Operations. Department of the Army, Washington, D.C.

Valente, A., Gil, Y., and Swartout, W. (1996). INSPECT: An intelligent system for air campaign plan evaluation based on EXPECT. Technical report, USC $\mathbf{Ð}$ Information Sciences Institute.

Van Creveld, M. L. (2000). The Art of War: War and Military Thought. Cassell, London.

Woodcock, A. E. R., Hitchins, D. K., and Cobb, L. (September, 2003). The strategic management system (STRATMAS) and the deployment of adaptable battle staffs. http://www.dodccrp.org/Proceedings/DOCS/wcd00000/wcd0005c.htm. 